\title{
PREVALÊNCIA DE TUMORES "DE NOVO" EM RECEPTORES DE TRANSPLANTE DE FÍGADO
}

\author{
"De Novo" post-transplant malignancies in liver transplant recipients \\ Ilka de Fátima Santana Ferreira Boin', Marilia Iracema Leonardi', \\ Ricardo Hoelz de Oliveira Barros', Jazon Romilson Almeida², Luiz Sergio Leonardi'
}

\begin{abstract}
RESUMO
0 risco de desenvolvimento de neoplasia após transplante hepático é de cerca de 1\% por ano, variando de 3 a 15\%, sendo, portanto, maior que o risco da população geral. A s causas potenciais de câncer após transplante de órgãos são: imunossupressão crônica, estimulação viral de linhas clonais celulares (sarcoma, câncer genital e hepatocelular). Objetivo: real izar uma revisão sobre o assunto e verificar a incidência de tumores "de novo" em nosso serviço. Métodos: Foram analisados retrospectivamente os prontuários de 325 casos de transplante de fígado realizados de setembro de 1991 a jul ho de 2006. Procurou-se observar a incidência desses tumores, analisando os tipos observados de malignidade, o tratamento efetuado e a sobrevida dos pacientes. Foi excluída a recidiva de carcinoma hepatocelular no fígado. Resultados: Foram observados cinco (1,54\%) casos: cinco homens com idade média de 49,2 anos. Observou-se $80 \%$ de mortalidade. A sobrevida deveuse à natureza do tumor ( pele), possi bilitando o tratamento cirúrgico adequado e o rastreamento feito por se tratar de paciente com a mesma manifestação anterior à cirurgia do transplante. Q uatro deles eram alcoolistas e em dois havia associação com tabagismo. Condusão: $\mathrm{A}$ alta mortalidade apresentada por aqueles doentes mostra a necessidade de um criterioso seguimento pós-operatório.
\end{abstract}

Descritores: Transplante Hepático, N eoplasia, I munossupressão.

Instituição:

1. Departamento de Cirurgia da Faculdade de Ciências Médicas da Universidade Estadual de Campinas e da Unidade de Transplante Hepático do Hospital de Clínicas da UNICAMP.

2. Departamento de Clínica Médica - Disciplina de Gastrenterologia e Médico do Gastrocentro - Universidade Estadual de Campinas.

Correspondência:

Ilka de Fatima Santana Ferreira Boin

Rua Aldo Oliveira Barbosa 184

CEP 13086-030 - Campinas - SP - Brasil

Tel. 5519 3289-1577

E-mail: ilka@fcm.unicamp.br

Recebido em: 10.07.2006

Aceito em: 25.07.2006

\section{INTRODUÇÃO}

0 transplante hepático é aceito como alternativa terapêutica desde 1983, devido a disponibilidade e confiabilidade dos imunossupressores desenvolvidos. ${ }^{1} 0$ risco de desenvolvimento de neoplasia após transplante hepático é de cerca de 1\% por ano, variando de três a $15 \%$, conforme o levantamento realizado, sendo, portanto, maior do que o risco da população geral.,3 0 câncer recorrente ou de novo é a segunda maior causa de mortalidade tardia nos transplantados hepáticos, estando atrás apenas das complicações cardiovasculares. ${ }^{4} \mathrm{~A}$ incidência aumentada de neoplasia naqueles pacientes reflete al guns fatores: seu perfil demográfico com fatores preexistentes de risco para câncer, infecções vi rais crônicas e ações dos imunossupressores exógenos. A maior parte das neoplasias que têm incidência aumentada nos transplantados está relacionada a infecções virais, como a doença linfoproliferativa associada ao vírus E pstein Barr e o câncer de pele, incluindo o câncer epidermóide e o sarcoma de $\mathrm{K}$ aposi, associado ao herpes vírus. ${ }^{5-8} \mathrm{O}$ objetivo deste trabal ho foi realizar uma revisão sobre o assunto, buscando obter informações para uma maior sobrevida aos pacientes submetidos a transplante hepático.

\section{CASUÍSTICA}

R etrospectivamente, foram analisados os prontuários de 325 casos de transplante ortotópico de fígado (TOF) realizados de setembro de 1991 a julho de 2006. 
Procurou-se observar a prevalência destes tumores, analisando-se os tipos observados de mal ignidade, idade dos pacientes, época do aparecimento do tumor, etiologia da doença hepática, tratamento efetuado e a sobrevida dos pacientes. Foi excluída a recidiva de carcinoma hepatocelular no fígado.

\section{RESULTADOS}

Foram observados cinco $(1,54 \%)$ casos, todos de homens com idade média de 49,2 anos. 0 tipo de tumor, idade de cada paciente, tempo de aparecimento da neoplasia após a realização do transplante e tempo de sobrevida de cada um deles após o transplante estão descritos na Tabela 1. Q uatro pacientes eram al cool istas, e dois deles eram tabagistas. A doença hepática que motivou o transplante foi cirrose hepática causada pelo vírus $C$, sendo que em quatro estava associada a alcoolismo. Observou-se $80 \%$ de mortalidade.

Tabela 1. Descrição do tipo de tumor, local de acometimento, idade e sexo do paciente, antecedente epidemiológico, tempo de aparecimento da neoplasia e de sobrevida após a realização do transplante de fígado.

\begin{tabular}{|c|c|c|c|c|c|c|}
\hline Tipo de tumor & Sexo & $\begin{array}{l}\text { Idade } \\
\text { (anos) }\end{array}$ & Antecedente & $\begin{array}{c}\text { Tempo } \\
\text { após TOF } \\
\text { (meses) }\end{array}$ & $\begin{array}{c}\text { SV } \\
\text { (meses) }\end{array}$ & $\begin{array}{c}\text { Estado } \\
\text { atual }\end{array}$ \\
\hline câncer de lábio & M & 57 & câncer de orelha & 72 & 84 & vivo \\
\hline $\begin{array}{l}\text { adenocarcinoma } \\
\text { de cólon }\end{array}$ & M & 39 & $\begin{array}{l}\text { alcoolismo/ } \\
\text { tabagismo }\end{array}$ & 48 & 108 & óbito \\
\hline $\begin{array}{l}\text { adenocarcinoma } \\
\text { de cólon }\end{array}$ & M & 53 & alcoolismo & 1 & 2 & óbito \\
\hline sarcoma de Kaposi & M & 54 & alcoolismo & 5 & 7 & óbito \\
\hline $\begin{array}{l}\text { carcinoma de } \\
\text { orofaringe }\end{array}$ & M & 43 & $\begin{array}{l}\text { alcoolismo/ } \\
\text { tabagismo }\end{array}$ & 24 & 42 & óbito \\
\hline
\end{tabular}

TOF = transplante de fígado

A sobrevida deveu-se ao tumor ser de pele (lábio). 0 paciente com tumor de cólon teve sobrevida de cinco anos por ter sido manifestação clínica por obstrução intestinal, o que possibilitou o tratamento cirúrgico adequado.

O outro paciente que apresentou tumor de cólon teve dois meses de sobrevida. 0 paciente com tumor de cabeça/pescoço foi submetido a radioterapia e cirurgia, e teve seis meses de sobrevida. 0 paciente com sarcoma de K aposi foi a óbito por hemorragia digestiva em dois meses de acompanhamento, após redução da imunossupressão.

\section{DISCUSSÃO}

O câncer, recorrente ou de novo é a segunda maior causa de mortalidadetardia nos transplantados hepáticos, estando atrás apenas das complicações cardiovasculares..$^{5,6,7}$ A incidência aumentada de neoplasia naqueles pacientes reflete alguns fatores: seu perfil demográfico com fatores preexistentes de risco para câncer, infecções virais crônicas e ações dos imunossupressores exógenos. ${ }^{8}$ A maior parte das neoplasias que tem incidência aumentada nos transplantados está relacionada a infecções virais, como a doença linfoproliferativa associada ao vírus E pstein-B arr, e câncer de pele, incluindo o câncer epidermóide e sarcoma de K aposi associado ao herpes vírus $8^{5,9}$. O utros trabal hos mostram claramentea importância da presença de fatores de risco para câncer antes do transplante, no desenvolvimento de neoplasias no seguimento desses pacientes. ${ }^{10,11}$
U m de nossos pacientes apresentou sarcoma de $\mathrm{K}$ aposi com pouco tempo de sobrevida e com antigenemia positiva para H H V-8.

O utro importante fator de risco para o desenvolvimento de neoplasia após transplante é a doença que causou a insuficiência hepática, sendo que a cirrose alcoólica aumenta muito a incidência de neoplasia, principalmente do tipo epidermóide. ${ }^{10}$ Numa série, a incidência aumentou de $5 \%$ para $26,7 \%$ entre os portadores de cirrose alcoólica. ${ }^{12}$

Vale a pena salientar que o esquema de imunossupressão escol hido não influencia a incidência de neoplasia após transplante hepático, ou seja, os esquemas imunossupressores mais novos não foram capazes de demonstrar menor incidência de neoplasia. ${ }^{13-15}$ Estudos com tempo de seguimento maior ainda são necessários para comprovar este aspecto.

Os tumores de pele são os mais comuns em pacientes submetidos a transplante hepático, somando cerca de $20 \%$ do total de tumores. ${ }^{14}$ A grande maioria deles ocorre em pacientes fumantes. Os fatores de risco são: idade, sexo mascul ino, cabelo ruivo, colangite esclerosante primária e uso de ciclosporina. ${ }^{15}$ Observamos somente um caso de câncer de lábio, e esse paciente já havia sido submetido a retirada de câncer de orelha antes do transplante.

O carcinoma epidermóide de orofaringe também está entre os mais freqüentes em transplantados hepáticos, com incidência aumentada comparada à população geral, mesmo comparada com população com hábitos de etilismo e tabagismo semel hantes, mostrando uma potencialização causada pelo esquema de imunossupressão. Em uma série de 171 pacientes, 12,2\% desenvolveram tumores epidermóides de orofaringe ou esôfago. ${ }^{12} 0$ risco em relação a população geral chega a ser 7,6 vezes maior ${ }^{16,17,18}$ Como citado, observamos um caso desse tipo de tumor associado ao tabagismo e ao alcoolismo.

Neoplasias da linhagem hematológica ocorrem em 1 a 3\% dos pacientes transplantados, podendo se manifestar como linfoma, leucemia ou como doença linfoproliferativa associada ao vírus E pstein B arr. ${ }^{1}$ E sta última vem mostrando melhores índices de sobrevivência com os novos esquemas de tratamento e imunossupressão, mas continua um importante problema a ser lidado no seguimento de pacientes transplantados. Não foi observado nenhum caso desse tipo de neoplasia. A maioria dos estudos não mostrou claramente aumento de incidência de câncer colorretal em pacientes submetidos a transplante hepático. A exceção são pacientes com pólipos no cólon anteriores ao transplante e, principalmente, pacientes cuja indicação ao transplante foi colangite esclerosante primária associada à retocolite ulcerativa.

Nesse subgrupo, a incidência de câncer colorretal chega a 6,5\% . Há uma clara necessidade de seguimento com colonoscopia e biópsia, e a necessidade de proctocolectomia profilática nesse subgrupo pode ser questionada. ${ }^{19,20}$ Foram observados dois pacientes com câncer de cólon nessa casuística. Um deles apareceu logo após o transplante, e não se pode afirmar se já havia lesão anterior. A manifestação clínica do outro foi obstrução intestinal (quatro anos após o transplante), possibilitando a ressecção intestinal e possibilitando uma sobrevida de 108 meses (60 meses após a ressecção intestinal).

\section{CONCLUSÃO}

A alta mortalidade apresentada por esses doentes mostra a necessidade de um seguimento pós-operatório criterioso, visando 0 diagnóstico e tratamento precoce dessas lesões. 


\begin{abstract}
Introduction: The risk of developing "de novo" malignancies after liver transplantation is around 1\% per year. The incidence varies from 3 to 15\%, therefore, being higher than that found among the general population. The potential causes for cancer af ter solid organ grafting are: chronic immunosuppression and human herpes viral infection. Purpose: The purpose of this paper was to review the medical literature on the incidence of "de novo" malignancies. Methods: A retrospective analysis of medical records of 325 patients submitted to orthotopic liver transplantation between September, 1991 and J uly, 2006 was performed. The type of tumor, risk factors involved, modality of treatment and the patient survival were recorded and analyzed. The recurrent hepatocellular carcinoma was excluded. Results: It was assessed $5(1.54 \%)$ males with mean age of 49.2 years. It was observed $80 \%$ of mortality. The survival was affected by the nature of the tumor (skin), which allowed adequate surgical treatment and due to the tracking performed, since that was a patient with the same manifestation previous to the transplant. Four of those patients were heavy alcohol consumers before the transplant associated to the consumption of tobacco and two of them presented associated consumption. Condusion: The high mortality presented by those patients shows the need of a criterious postsurgical follow-up.
\end{abstract}

Keywords: Transplantation, Liver, M alignancy I munossupression, Incidence

\section{REFERENCES}

1. Jain A, Nalesnik M, Reyes J, Pokharna R, Mazariegos G, Green M, et al. Posttransplant lymphoproliferative disorders in liver transplantation: a 20-year experience. Ann Surg. 2002, Oct;236(4):429-37.

2. Xiol X, Guardiola J, Menendez S, Lama C, Figueras J, Marcoval J, et al., Risk factors for development of de novo neoplasia after liver transplantation, Liver Transplant. 2001 Nov;7(11):971-5.

3. Haagsma EB, Hagens VE, Schaapveld M, van den Berg AP, de Vries EG, Klompmaker IJ, et al. Increased cancer risk after liver transplantation: a population-based study, J Hepatol. 2001 Jan;34(1):84-91.

4. Galve ML, Cuervas-Mons V, Figueras J, Herrero I, Mata M, Clemente G, et al. Incidence and outcome of de novo malignancies after liver transplantation, Transplant Proc. 1999 Feb-Mar;31(1-2):1275-7.

5. Fung JJ, Jain A, Kwak EJ, Kusne S, Dvorchik I, Eghtesad B, De novo malignancies after liver transplantation: a major cause of late death, Liver Transplant. 2001 Nov;7(11 Suppl 1):S109-18.

6. Vogt DP, Henderson JM, Carey WD, Barnes D, The long-term survival and causes of death in patients who survive at least 1 year after liver transplantation, Surgery. 2002 Oct;132(4):775-80;

7. Sheiner PA, Magliocca JF, Bodian CA, Kim-Schluger L, Altaca G, Guarrera $\mathrm{JV}$, et al., Long-term medical complications in patients surviving $>$ or $=5$ years after liver transplant, Transplantation. 2000 Mar 15;69(5):781-9.

8. Sheil AG, Malignancy following liver transplantation: a report from the Australian Combined Liver Transplant Registry, Transplant Proc. 1995 Feb;27(1):1247.

9. Niedobitek G, Mutimer DJ, Williams A, Whitehead L, Wilson P, Rooney N, et al. Epstein-Barr virus infection and malignant lymphomas in liver transplant recipients, Int J Cancer. 1997 Nov 14;73(4):514-20.

10. Menachem Y, Safadi R, Ashur Y, Ilan Y, Malignancy after liver transplantation in patients with premalignant conditions [In Process Citation], J Clin Gastroenterol. 2003 May-Jun;36(5):436-9.

11. Menachem Y, Safadi R, Ashur Y, Eid A, Jurim O, Ilan Y, Premalignant conditions: risk factor for postliver transplantation malignancy, Transplant Proc. 2001 Sep;33(6):2935-6.

12. Duvoux C, Delacroix I, Richardet JP, Roudot-Thoraval F, Metreau JM, Fagniez PL, et al. Increased incidence of oropharyngeal squamous cell carcinomas after liver transplantation for alcoholic cirrhosis, Transplantation. 1999 Feb 15;67(3):418-21.

13. Jonas S, Rayes N, Neumann U, Neuhaus R, Bechstein WO, Guckelberger $\mathrm{O}$, et al. De novo malignancies after liver transplantation using tacrolimusbased protocols or cyclosporine-based quadruple immunosuppression with an interleukin-2 receptor antibody or antithymocyte globulin, Cancer. 1997 Sep 15;80(6):1141-50.

14. Euvrad S, Kanitakis J. Skin cancers fter liver transplantation: what to do? J Hepatol. 2006;44(1):27-32.

15. Mithoefer AB, Supran S, Freeman RB, Risk factors associated with the development of skin cancer after liver transplantation, Liver Transplant. 2002 Oct;8(10):939-44.

16. Jain AB, Yee LD, Nalesnik MA, Youk A, Marsh G, Reyes J, et al. Comparative incidence of de novo nonlymphoid malignancies after liver transplantation under tacrolimus using surveillance epidemiologic end result data. Transplantation. 1998, 15;66(9):1193-200.

17. Saigal S, Norris S, Muiesan P, Rela M, Heaton N, O'Grady J, Evidence of differential risk for posttransplantation malignancy based on pretransplantation cause in patients undergoing liver transplantation, Liver Transplant. 2002 May;8(5):482-7.

18. Duvoux C, Cherqui D, Delacroix I, Metreau JM, Fagniez PL, Dhumeaux D, Upper respiratory and digestive tract malignancies after liver transplantation for alcoholic cirrhosis. Transplant Proc. 1996 Oct;28(5):2883.

19. Carson KL, Hunt CM, Medical problems occurring after orthotopic liver transplantation, Dig Dis Sci. 1997 Aug;42(8):1666-74.

20. Higashi H, Yanaga K, Marsh JW, Tzakis A, Kakizoe S, Starzl TE. Development of colon cancer after liver transplantation for primary sclerosing cholangitis associated with ulcerative colitis, Hepatology. 1990 Mar;11(3):477-80. 